\title{
Educação em saúde e busca ativa de casos de hanseníase em uma escola pública em Ananindeua, Pará, Brasil
}

\author{
Health education and active search for cases of leprosy in a public school in Ananindeua, Pará, Brazil
}

\author{
Educación para la salud y búsqueda activa de casos de lepra en una escuela pública en \\ Ananindeua, Pará, Brasil
}

\author{
Bruna Ranyelle Marinho Sousa. Universidade Federal do Pará (UFPA). bruna-ranyelle@hotmail.com (Autora correspondente) \\ Francisco Hepaminondas Abreu Moraes. Universidade Federal do Pará (UFPA). hepaminondasmoraes@hotmail.com \\ Jocyane Souza Andrade. Universidade Federal do Pará (UFPA). cyanny_10@yahoo.com.br \\ Ennye Sakaguchi Lobo. Hospital Pronto Socorro Municipal Mário Pinotti, Belém (PA). enalawolf@hotmail.com \\ Enerli Araújo Macedo. Prefeitura Municipal de Ananindeua (PA). henerli@hotmail.com \\ Carla Andréa Avelar Pires. Universidade Federal do Pará (UFPA). carlaavelarpires@bol.com.br \\ Egon Luiz Rodrigues Daxbacher. Universidade Estadual do Rio de Janeiro (UERJ). egondax@gmail.com
}

\section{Resumo}

Objetivo: Descrever ação para o controle da hanseníase por meio da educação em saúde e da busca ativa de casos na faixa etária escolar, em uma instituição pública de ensino do município de Ananindeua, Pará. Métodos: Trata-se de estudo descritivo, conduzido em uma instituição pública de ensino, incluindo estudantes do ensino fundamental. Em outubro e novembro de 2010 foram realizadas palestras na escola sobre hanseníase e outras dermatoses e, em seguida, foi feita uma triagem com os alunos que apresentaram alguma mancha na pele. No total de 532 alunos envolvidos, foram identificados 55 alunos como casos suspeitos para hanseníase. Esses alunos foram conduzidos para consulta dermatológica, sendo realizados o exame clínico e a aplicação de questionários sociodemográficos. Resultados: A face (49\%) e os membros superiores (45\%) foram as principais regiões do corpo com lesões. Na maioria dos casos investigados tratava-se de pitiríase alba (42\%) e pitiríase versicolor (31\%). Entretanto, diagnosticou-se um caso de hanseníase (2\%), na forma clínica tuberculóide. Conclusão: Considerando a abrangência do estudo, o número de casos de hanseníase encontrado é significativo e reafirma a alta taxa de detecção de hanseníase em menores de 15 anos no município. Ações de vigilância epidemiológica devem ser intensificadas, no sentido de detectar a doença mais precocemente, contribuindo para a redução de estigmas e da restrição à participação social.

\section{Abstract}

Objectives: To describe action for leprosy control through health education and case active search among school age children in a public school in the municipality of Ananindeua, Pará state. Methods: This is a descriptive study conducted in a public school including elementary school students. In October and November 2010, lectures on leprosy and other skin lesions were given in this school and then screening was performed with students that presented skin spots. From the total of 532 students involved, 55 were identified as suspected to leprosy. These students were taken to dermatological appointment including clinical examination and application of socio-demographic questionnaire. Results: The face (49\%) and upper limbs (45\%) were the main body areas presenting lesions. In most of the cases investigated, pityriasis alba (42\%) and pityriasis versicolor (31\%) were diagnosed. However, one case of leprosy (2\%) was also found, in tuberculoid clinical form. Conclusion: Considering the scope of the study, the number of leprosy cases found is significant and confirms the high detection rate of leprosy in children under age 15 in the city. Epidemiological surveillance actions should be intensified in order to detect the disease in its early stage, contributing to enhancement in social participation by reducing the disease-related stigma.

\section{Resumen}

Objetivo: Describir la acción para el control de la lepra mediante la Educación para la Salud y la búsqueda activa de casos en niños en edad escolar en una escuela pública en la ciudad de Ananindeua, Pará. Métodos: Se trata de un estudio descriptivo, realizado en una escuela pública, con los estudiantes de primaria. En octubre y noviembre de 2010 se realizaron conferencias sobre lepra y otras enfermedades de la piel y luego se examinó a los estudiantes que tenían alguna mancha en la piel. Del total de 532 alumnos, 55 estudiantes fueron identificados como sospechosos de tener lepra. Esos estudiantes fueron derivados a consulta dermatológica, examen clínico y aplicación de cuestionarios socio-demográficos. Resultados: La cara (49\%) y las extremidades superiores (45\%) fueron las principales áreas del cuerpo con lesiones. En la mayoría de los casos se constató la pitiriasis alba (42\%) y pitiriasis versicolor (31\%). Sin embargo, se diagnosticó un caso de lepra (2\%), en la forma clínica tuberculoide. Conclusión: Teniendo en cuenta el alcance del estudio, el número de casos de lepra encontrado es significativo y reafirma la alta tasa de detección de lepra en los niños menores de 15 años en la ciudad. Las acciones de vigilancia epidemiológica deben ser intensificadas, con el fin de detectar precozmente la enfermad, contribuyendo para la reducción del estigma y de la restricción de la participación social.

Como citar: Sousa BRM, Moraes FHA, Andrade JS, Lobo ES, Macedo EA, Pires CAA, et al. Educação em saúde e busca ativa de casos de hanseníase em uma escola pública em Ananindeua, Pará, Brasil. Rev Bras Med Fam Comunidade. 2013;8(27):143-9. Disponível em: http://dx.doi.org/10.5712/rbmfc8(27)467
Palavras-chave: Hanseníase Mycobacterium leprae Adolescentes Educação em Saúde Estudos Transversais

Keywords: Leprosy Mycobacterium leprae Adolescent Health Education Cross-sectional Studies

Palabras clave:

Lepra

Mycobacterium leprae Adolescentes Educación en Salud Estudios Transversales 


\section{Introdução}

A hanseníase é uma doença infecto-contagiosa causada pela Mycobacterium leprae. Apresenta evolução crônica, atingindo predominantemente a pele e os nervos periféricos. É considerada potencialmente incapacitante e, embora curável, seu diagnóstico ainda causa grande impacto psicossocial e comprometimento da qualidade de vida ${ }^{1,2}$. Trata-se de uma doença endêmica no Brasil, o que torna o país responsável por $93 \%$ dos casos de hanseníase no continente americano. O Brasil apresentou em 2011 coeficiente geral de detecçáo de novos casos de 17,65/100.000 habitantes. Nesse ano foram diagnosticados 33.955 casos novos, sendo 2.420 (7,12\%) em menores de 15 anos. Segundo o Ministério da Saúde, observou-se ainda que 2.165 pacientes apresentaram grau II de incapacidade ${ }^{3,4}$.

No estado do Pará, o coeficiente geral de detecção de novos casos na faixa etária de menores de 15 anos foi de 18,29/100.000 habitantes, sendo classificado como hiperendêmico e revelando a persistência na transmissão da doença e as dificuldades dos programas de saúde para o controle desse agravo ${ }^{4,5}$.

O município de Ananindeua, segundo mais populoso do Pará, é um dos 87 municípios do estado com situação hiperendêmica para hanseníase em crianças e adolescentes. Segundo dados do Sistema de Informação de Agravos de Notificação (SINAN), fornecidos pela Secretaria de Vigilância Epidemiológica de Ananindeua, o coeficiente de detecção de novos casos foi 6/100.000 em 2008 e, apesar da redução de 45\% em relação a 2004, as políticas de controle de hanseníase precisam continuar e se intensificar. Entre os menores de 15 anos com hanseníase, a faixa etária com maior quantidade de casos é a de 10 a 14 anos. Segundo o SINAN, a hanseníase mostrou-se mais incidente em homens, 57\% em 2008, sendo que a forma clínica de notificação mais acometida foi a dimorfa e que $69 \%$ dos casos foram multibacilares ${ }^{6}$.

Para a transmissão da hanseníase, é necessário o convívio prolongado com pacientes transmissores do bacilo de Hansen. Tais bacilos são eliminados pelas secreçôes nasais e da orofaringe dos indivíduos multibacilares e penetram no organismo do indivíduo sadio pelas vias aéreas superiores ${ }^{7}$. Estudos relatam a participação de fatores genéticos contribuindo para a infecção e fenótipos clínicos da hanseníase ${ }^{8}$.

A doença, inicialmente, pode se manifestar por meio de lesôes de pele, tais como máculas hipocrômicas ou avermelhadas que apresentam alteração de sensibilidade. Essas lesôes ocorrem em qualquer região do corpo, mas com maior frequência na face, orelhas, nádegas, braços, pernas e costas. Podem aparecer nervos espessados e doloridos, diminuição de sensibilidade nas áreas inervadas, resultando em comprometimento sensitivo, motor e autonômico (alteraçóes de glândulas sudoríparas e sebáceas) responsáveis pelas incapacidades e deformidades características da hanseníase 9 . O diagnóstico é essencialmente clínico, baseado nos sinais e sintomas, no exame da pele (avaliação dermatoneurológica) e dos nervos periféricos e na história epidemiológica. Excepcionalmente há necessidade de auxílio laboratorial para a confirmação diagnóstica, sendo que seu diagnóstico é de competência da atenção primária à saúde ${ }^{7}$.

A hanseníase é uma enfermidade considerada de adultos devido ao seu longo período de incubação, entretanto, em áreas endêmicas e quando ocorrem casos na família, o risco de crianças adoecerem aumenta. A ocorrência de hanseníase em menores de 15 anos reflete a exposição precoce e intensa, com alta carga bacilar. Dessa forma, a ocorrência de hanseníase em crianças é considerada um indicador da prevalência da doença na população geral e sua detecção é importante, uma vez que indica a necessidade de atividades de vigilância epidemiológica para busca de contactantes e identificação do caso fonte ${ }^{10,11}$.

O Programa Nacional de Controle de Hanseníase (PNCH) tem como prioridade a reduçáo de casos em menores de 15 anos de idade, pois esses casos têm relação com doença recente e focos de transmissão ativos. Seu monitoramento epidemiológico é de grande relevância para o controle da hanseníase ${ }^{12}$. Na Estratégia Global para Redução Adicional da Carga da Hanseníase quinquênio de 2011 a 2015, da Organização Mundial da Saúde, reiterou-se que o controle da hanseníase continuará a depender da deteç̧ão precoce e do tratamento com a poliquimioterapia (PQT) ${ }^{13}$.

Nesse sentido, é de grande importância que no estado do Pará, especialmente na cidade de Ananindeua, sejam exercidas ações visando o diagnóstico precoce, tais como a busca ativa de casos, além de açôes de educação em saúde para o maior controle da hanseníase, principalmente na faixa etária escolar, na qual os índices de detecção são altos, indicativos de focos ativos de transmissão.

Nesse contexto, esse estudo teve como objetivo contribuir para o controle da hanseníase por meio da educação em saúde e da busca ativa de casos na faixa etária escolar em uma escola pública do município de Ananindeua, estado do Pará, fornecendo informaçóes e realizando exame dermatológico a fim de diagnosticar precocemente os casos de hanseníase e de outras dermatoses nessa população. 


\section{Metodologia}

Trata-se de estudo descritivo, tendo como base populacional estudantes do ensino fundamental ( $6^{\circ}$ ao $9^{\circ}$ ano) de uma escola municipal localizada no município de Ananindeua, Pará, no Conjunto Stélio Maroja. O estudo foi realizado nos meses de outubro e novembro de 2010 e consistiu de duas etapas: educação em saúde na escola e consulta dos alunos selecionados.

Inicialmente foram realizadas atividades de educação em saúde com os 532 alunos matriculados no ensino fundamental (60 ao $9^{\circ}$ ano), por meio de palestras ministradas de sala em sala, nos turnos da manhã e da tarde, com o objetivo de orientar e informar os estudantes sobre a hanseníase, a importância do diagnóstico precoce e o tratamento. Outras doenças dermatológicas foram também abordadas, com o intuito de realizar o diagnóstico diferencial com a hanseníase. Durante esse primeiro momento, cada aluno recebeu uma ficha para preenchimento de informaçóes sobre dados pessoais e sociodemográficos que deveria ser preenchida caso o entrevistado relatasse a presença de alguma alteraçáo na pele. Ao final das palestras, foram selecionados os alunos que apresentavam mácula hipocrômica ou eritematosa ou outra lesão sugestiva de hanseníase e que aceitaram participar da pesquisa.

Dessa forma, a população do estudo foi composta por 55 (cinquenta e cinco) alunos que inicialmente preencheram um questionário sociodemográfico e que posteriormente foram encaminhados para a avaliação dermatoneurológica realizada por uma médica dermatologista, constituindo-se na segunda etapa do projeto. Os pais e/ou responsáveis assinaram Termos de Consentimento Livre e Esclarecido (TCLE) permitindo a participação dos menores no estudo.

As crianças com problemas dermatológicos simples identificados receberam prescrição durante a realização da consulta com a médica especializada. Aquelas identificadas como portadoras de hanseníase foram encaminhadas à unidade básica de saúde do bairro para realizar tratamento específico.

Importante ressaltar que os professores e a diretora se mostraram muito solícitos durante a realização da pesquisa, compreendendo a importância do estudo e contribuindo para o seu desenvolvimento, disponibilizando parte do tempo de aula para que fossem realizadas as palestras sobre hanseníase e demais alteraçóes dermatológicas. Os pais e/ou responsáveis, entretanto, apesar de terem assinado o TCLE e terem sido esclarecidos sobre a importância da consulta dermatológica, náo se mostraram muito ativos e interessados nos resultados que seriam obtidos com a realização do estudo.

Os resultados obtidos foram armazenados em banco de dados para serem analisados e demonstrados por meio de tabelas e figuras, utilizando o software Microsoft Office Excel 2007.

O presente trabalho seguiu as recomendaçóes do Conselho Nacional de Ética em Pesquisa (CONEP), sendo aprovado pelo Comitê de Ética e Pesquisa (CEP) do Instituto de Ciências da Saúde da Universidade Federal do Pará (ICS-UFPA), CAAE 3167.0.000.073-10 e parecer no 112/10.

\section{Resultados}

O perfil sociodemográfico dos alunos selecionados para a consulta dermatológica (Tabela 1) mostrou predominância do gênero feminino, faixa etária entre 12 e 14 anos incompletos e cor parda. Com relação à escolaridade, o número de alunos por ano foi bem distribuído, sendo a maioria dos alunos atendidos estudantes do $6^{\circ}$ ano e do turno da manhã.

Quanto à procedência (Tabela 2), verificou-se que a maioria dos alunos atendidos pela pesquisa era da localidade Nova Esperança, situada no bairro do Coqueiro, próximo ao Conjunto Stélio Maroja.

De acordo com o Figura 1, verifica-se que, conforme relato dos entrevistados, as principais regióes do corpo que apresentavam as lesôes foram: a face (49\%), os membros superiores (45\%) e o tórax (33\%).

Com a realização da consulta dermatológica foi possível estabelecer o diagnóstico das manchas apresentadas pelos alunos (Figura 2). 
Tabela 1. Perfil sociodemográfico dos 55 alunos atendidos por consulta dermatológica no município de Ananindeua, Pará, em 2010.

\begin{tabular}{lcc}
\hline \multicolumn{1}{c}{ Variáveis } & Alunos (n) & Alunos (\%) \\
\hline Gênero & & \\
Masculino & 20 & 36 \\
Feminino & 35 & 64 \\
Idade & & \\
10 I-12 & 15 & 27 \\
12 I-14 & 19 & 35 \\
14 I-16 & 16 & 29 \\
+ de 16 & 05 & 09 \\
Raça & & \\
Branca & 13 & 24 \\
Parda & 26 & 47 \\
Negra & 07 & 13 \\
Indígena & 01 & 02 \\
Não declarou & 08 & 14 \\
Série do ensino fundamental & & \\
$6^{0}$ ano & 18 & 33 \\
$7^{0}$ ano & 12 & 22 \\
$8^{0}$ ano & 11 & 20 \\
$9^{0}$ ano & 14 & 25 \\
Turno & & 60 \\
Manhã & 33 & 40 \\
\hline Tarde & 22 & \\
\hline
\end{tabular}

Tabela 2. Procedência dos alunos que foram atendidos na consulta dermatológica no município de Ananindeua, Pará, em 2010.

\begin{tabular}{lcc}
\hline \multicolumn{1}{c}{ Localidades de Ananindeua } & Alunos (n) & Alunos (\%) \\
\hline Conjunto Cidade Nova & 09 & 16 \\
40 Horas & 07 & 13 \\
Nova Esperança & 19 & 34 \\
Conjunto Stélio Maroja & 07 & 13 \\
Loteamento 28 de Agosto & 05 & 09 \\
Outros & 08 & 14 \\
\hline
\end{tabular}

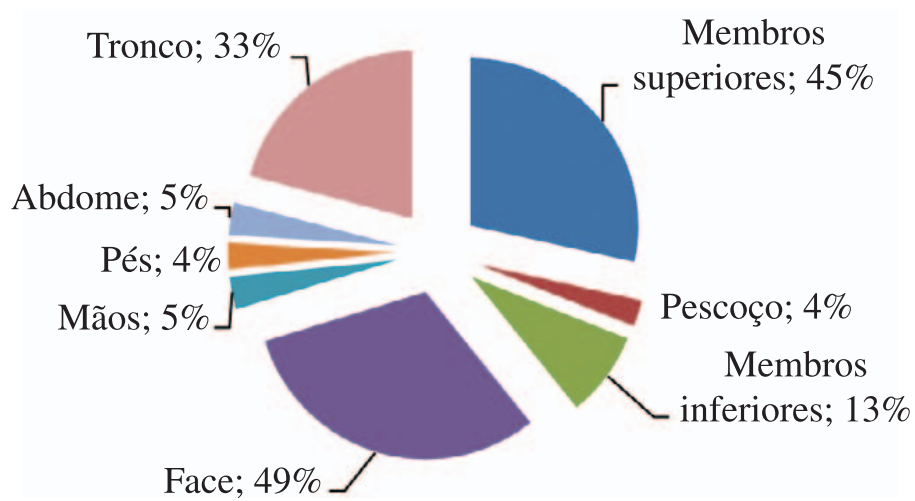

Figura 1. Principais regiões do corpo que apresentavam lesões nos alunos entrevistados no município de Ananindeua, Pará, em 2010. 


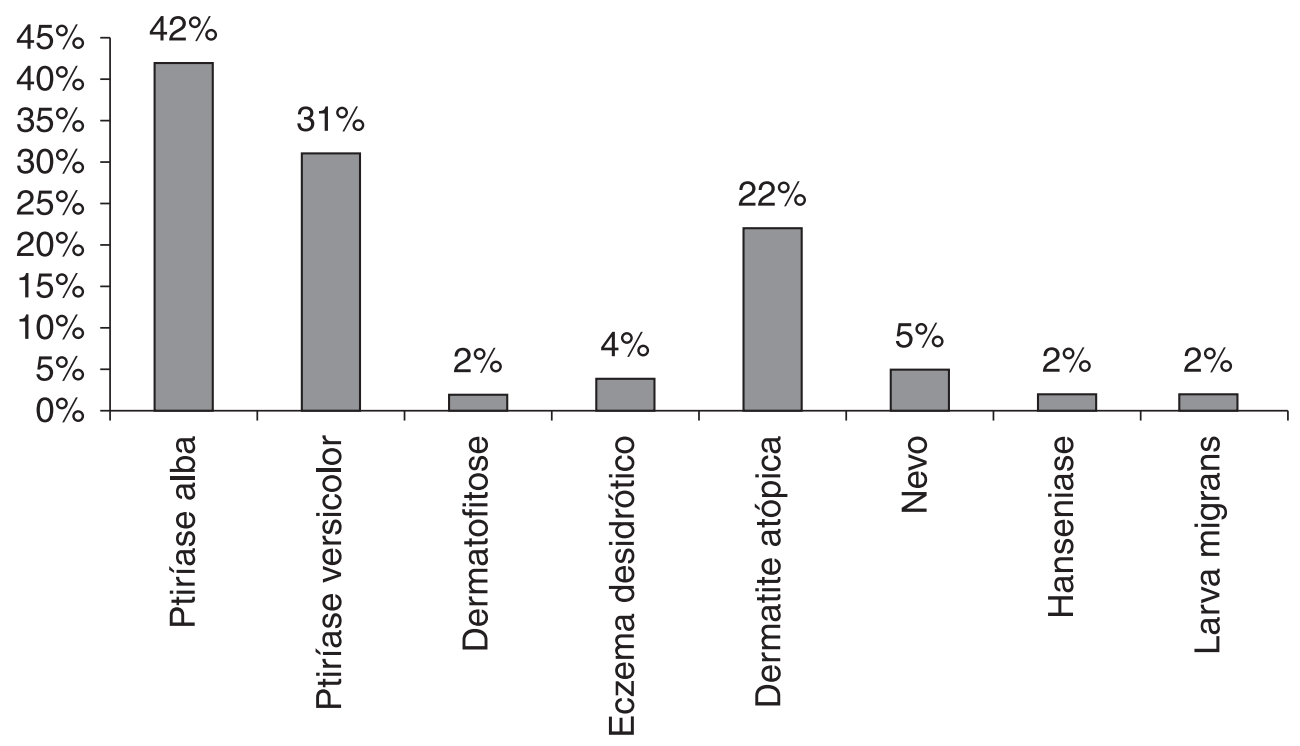

Figura 2. Diagnósticos encontrados nos alunos avaliados em consulta dermatológica no município de Ananindeua, Pará, em 2010.

Observou-se que a maioria dos alunos apresentou pitiríase alba (42\%), pitiríase versicolor (31\%) e dermatite atópica (22\%). Além disso, o estudo permitiu o diagnóstico precoce de um caso de hanseníase, na forma clínica tuberculóide, paucibacilar. O perfil do caso encontrado era: sexo masculino, pardo, 15 anos, $7^{\text {a }}$ série, morador do Loteamento 28 de Agosto. A lesão apresentava mácula hipocrômica com bordas papulosas, irregulares e limites imprecisos localizada em sua mão direita, além de placa eritematosa no cotovelo direito, com alteração de sensibilidade térmica e espessamento do nervo ulnar. Essa criança foi encaminhada à unidade básica de saúde mais próxima de sua residência e iniciou tratamento com poliquimioterapia para hanseníase. Foi realizada busca ativa de casos no domicílio, náo tendo sido encontrando nenhum caso ativo, embora houvesse histórico familiar de hanseníase já tratada e curada.

Durante as consultas do estudo, identificou-se ainda outro aluno, do sexo masculino, pardo, morador da área de ocupação Nova Esperança, que já teve a doença e concluiu o tratamento, apresentando apenas lesóes residuais na pele. Importante salientar que as crianças não tiveram seu diagnóstico revelado para os colegas, não sendo expostas a nenhum tipo de preconceito.

\section{Discussão}

Os dados encontrados sobre hanseníase são significativos dentro da população estudada, considerando-se os coeficientes utilizados pelo Programa Nacional de Controle da Hanseníase (PNCH). O estado do Pará apresenta coeficiente de detecção de casos novos de hanseníase em menores de 15 anos de 18,29/100.000 habitantes, segundo dados de 20115. Nesse estudo, encontrou-se um caso de hanseníase em uma amostra de 532 alunos, o que evidencia uma taxa bastante alta, muito maior que os dados atuais apresentados para o estado do Pará, refletindo a condição hiperendêmica do município. Entretanto, é necessário considerar que tal discrepância talvez se deva à amostra bastante reduzida do estudo.

A redução de casos em menores de 15 anos de idade, como o caso observado nesse estudo, é prioridade do PNCH, uma vez que esses casos têm relação com doença recente e focos de transmissão ativos e seu monitoramento epidemiológico é relevante para o controle da hanseníase ${ }^{12}$.

Em um estudo semelhante feito por Silva et al. ${ }^{14}$ em estudantes de escolas públicas no município de Buriticupu, estado do Maranhão, foram identificados 20 casos de hanseníase entre 14.653 estudantes, o que representa uma taxa de detecção de 1,36 casos para cada 1.000 indivíduos. Entre as faixas etárias observadas, a maior detecção de casos ocorreu entre 11 anos e 14 anos, com um total de 10 casos de hanseníase. Esse estudo apresenta dados semelhantes aos observados na presente pesquisa, que apresentou taxa de detecção de casos de 1,87/1.000. 
A detecção de casos de hanseníase no sexo masculino, forma paucibacilar e com presença de histórico familiar nessa casuística está em consonância com o estudo de Lastória e Abreu ${ }^{15}$, que realizaram busca ativa de casos de hanseníase no estado de São Paulo. Eles encontraram um índice de detecção de 4\% em menores de 15 anos, superior ao do presente estudo, provavelmente por limitação de amostra.

Em estudo realizado por Ferreira et al. ${ }^{16}$ sobre dermatoses pediátricas, observou-se maior prevalência de dermatoses alérgicas $(28,0 \%)$, seguidas por dermatoses inflamatórias $(18,6 \%)$, dermatoses pigmentares $(15,9 \%)$ e dermatoses infecciosas $(14,4 \%)$. Em contrapartida, no presente estudo a maior prevalência foi de pitiríase alba (42\%) e pitiríase versicolor $(31 \%)$, assim como as dermatoses alérgicas representaram $22 \%$ dos casos. Laczynski e Cestari ${ }^{17}$ encontraram a pitiríase alba entre as principais dermatoses em crianças, principalmente de escola pública, o que corrobora dados do presente estudo.

Um fato importante a ser discutido é a procedência dos casos diagnosticados pelo estudo. A maioria dos alunos era da localidade Nova Esperança (34\%), Conjunto Cidade Nova (16\%) e Conjunto Stélio Maroja (13\%). A área de ocupação Nova Esperança é uma localidade que faz parte do Bairro do Coqueiro, em Ananindeua, constituindo uma área de condiçôes higiênico-sanitárias precárias e que, de acordo com a investigação, apresentou um caso de hanseníase já tratada. O Conjunto Stélio Maroja é constituído em sua grande maioria por residências mal arejadas, pequenas e bastante próximas umas das outras, o que pode facilitar a transmissibilidade da hanseníase, bem como de outras doenças, tais como tuberculose e pneumonia. O caso ativo de hanseníase encontrado provém do Loteamento 28 de Agosto, que apresenta condiçóes precárias semelhantes às do Conjunto Nova Esperança. Esses fatores são relevantes para a monitorizarão de áreas de risco e para a implementação de medidas de controle e planejamento das açóes de vigilância em saúde.

\section{Conclusão}

O número de casos de hanseníase encontrados pode aparentemente ser irrelevante, no entanto, considerando o pequeno tamanho amostral, o resultado torna-se significativo, reafirmando a alta detecção de hanseníase em menores de 15 anos no município, principalmente na faixa escolar. Isso revela a intensidade de transmissão da doença, alertando para o fato de que muitos outros casos podem estar ocultos na própria família ou localidade.

Dessa forma, verifica-se a importância de proporcionar melhores condições de vida e moradia aos indivíduos com o objetivo de interromper a cadeia de transmissibilidade, além do monitoramento de áreas endêmicas e aumento de açôes educativas e preventivas, a fim de se detectar a doença mais precocemente e evitar as consequências do diagnóstico tardio e dos estigmas sociais. Observa-se, assim, a necessidade de maiores estudos, especialmente nessa faixa etária que se apresenta como importante marcador de transmissáo da hanseníase.

Convém ressaltar que o estudo promoveu como benefício a educação em saúde para os alunos no que tange à hanseníase e outras dermatoses, permitindo também que muitas dessas fossem diagnosticadas e tratadas. A alta incidência de pitiríase alba nos alunos, bem como a busca ativa de casos intradomiciliares de comunicantes de hanseníase, podem ser alvo de estudos futuros.

\section{Referências}

1. Azulay DR, Azulay RD. Hanseníase. In: Azulay RD, Azulay DR. Dermatologia. Rio de Janeiro: Guanabara Koogan; 1999. cap. 19 , p. $223-233$.

2. Martins BDL, Torres FN, Oliveira MLW. Impacto na qualidade de vida em pacientes com hanseníase: correlação do Dermatology Life Quality Index com diversas variáveis relacionadas à doença. An Bras Dermatol. 2008; 83(1): 39-43. http://dx.doi.org/10.1590/S0365-05962008000100005

3. Barbiere CLA, Marques HHS. Hanseníase em Crianças e Adolescentes: Revisão Bibliográfica e Situação Atual no Brasil. Pedriatria. 2009; 31(4); $281-290$.

4. Brasil. Ministério da Saúde. Sistema de Informação de Agravos Notificáveis/SINAN. Registro ativo: número e percentual. Casos novos de hanseníase: número, coeficiente e percentual, faixa etária, classificação operacional, sexo, grau de incapacidade, contatos examinados, por estados e regiões. Brasília: SINAN; 2011. [acesso em 2013 Mar 20] Disponível em: http://portal.saude.gov.br/portal/arquivos/pdf/indi_operacionais_epimieologicos_ hans_br_2011.pdf.

5. Brasil. Ministério da Saúde. Sistema de Informação de Agravos Notificáveis/SINAN. Coeficientes de prevalência, detecção geral e em menores de 15 anos de Hanseníase por Região. Brasília: SINAN; 2011. [acesso em 2013 Mar 20] Disponível em: http://portal.saude.gov.br/portal/arquivos/pdf/ coef_prev_detec_geral_menor_15_hans_reg_br2011.pdf. 
6. Brasil. Ministério da Saúde. Sistema de Informação de Agravos Notificáveis/SINAN. Casos novos de Hanseníase notificados no município de Ananindeua, Pará, no período de 2004 a 2008. Brasília: SINAN. [acesso em 2010 Maio 26] Disponível em: http://portal.saude.gov.br/portal/arquivos/ pdf/boletim_novembro.pdf.

7. Gallo MEN, Sampaio E, Nery JAC, Moraes MO, Antunes SL, Ressolani MCV, et al. Hanseníase: Aspectos Epidemiológicos, Clínicos e Imunológicos. In: Coura JR. Dinâmicas das Doenças Infecciosas e Parasitárias, v. 2. Rio de Janeiro: Guanabara koogan; 2005. cap. 116, p. 1383-1392.

8. Prevedello FC, Mira MT. Hanseníase: uma doença genética? An Bras Dermatol. 2007; 82(5): 451-9. http://dx.doi.org/10.1590/S036505962007000500009

9. Vieira CSCA, Soares MT, Ribeiro CTSX, Silva LFG. Avaliação e controle de contatos faltosos de doentes com Hanseníase. Rev Bras de Enferm. 2008; 61(número especial): 684-5. http://dx.doi.org/10.1590/S0034-71672008000700005

10. Imbiriba EB, Hurtado-Guerrero JC, Garnelo L, Levino A, Cunha MG, Pedrosa V. Perfil epidemiológico da hanseníase em menores de quinze anos de idade, Manaus (AM), 1998-2005. Rev Saúde Pública. 2008; 42(6): 1021-1026. http://dx.doi.org/10.1590/S0034-89102008005000056

11. Souza VFM, Valle CLP, Daxbacher ELR, Silva RS, Obadia DL. Relato de três casos novos de hanseníase em menores de quinze anos no município de Itaguaí, Rio de Janeiro: evento de alerta para investigação epidemiológica. An Bras Dermatol. 2011: 86(5): 1011-1015. http://dx.doi.org/10.1590/ S0365-05962011000500024

12. Brasil. Ministério da Saúde. Saude.gov.br [página da Internet]. Política Nacional de Controle da Hanseníase. [acesso em 2010 Maio 26]. Disponível em:http://portal.saude.gov.br.

13. World Health Organization - WHO. Global Strategy for furtherreducing the disease burden due to leprosy: plan period: 2011-2015. Geneva: WHO; 2010.

14. Silva AR, Portela EGL, Matos WB, Silva CCB, Gonçalves EGR. Hanseníase no município de Buriticupu, Estado do Maranhão: busca ativa na população estudantil. Rev Soc Bras Med Trop. 2007; 40(6): 657-60. http://dx.doi.org/10.1590/S0037-86822007000600012

15. Lastória JC, Morgado de Abreu MAM. SBD-RESP na busca ativa de casos de hanseníase. An Bras Dermatol. 2011; 86(3): 613-20. http://dx.doi. org/10.1590/S0365-05962011000300039

16. Ferreira FR, Nascimento LFC, D, Cirvidiu DC. Prevalência de dermatoses pediátricas em um Hospital Universitário na região sudeste do Brasil. An Bras Dermatol. 2011; 86(3): 477-82. http://dx.doi.org/10.1590/S0365-05962011000300009

17. Laczynski CMM, Cestari SCP. Prevalência de dermatoses em escolares na região do ABC paulista. An Bras Dermatol. 2011; 86(3): 469-76. http:// dx.doi.org/10.1590/S0365-05962011000300008 